\title{
Chain Dynamics of Polystyrene in High Viscosity Solvents Studied by the Fluorescence Depolarization Method
}

\author{
Keiko Ono, Yasuo OKada, Syunsuke YoKotsuka, \\ Shinzaburo Iто, and Masahide Yамамото* \\ Division of Polymer Chemistry, Graduate School of Engineering, \\ Kyoto University, Kyoto 606, Japan
}

(Received August 2, 1993)

\begin{abstract}
Polystyrene (PS) labeled with anthracene in the middle of the main chain was prepared, and the relaxation time of their local motion in the solvents with various viscosities was examined by the fluorescence depolarization technique. We measured the relaxation time of emission anisotropy, $T_{m}$, in four kinds of mixed solvents of diethylphthalate (DEP) and cyclohexanone (CHO). These are good solvents for PS and have different viscosities. With the addition of DEP to cyclohexanone, i.e., with the increase in solvent viscosity, the activation energy of $T_{\mathrm{m}}, E^{*}$, obtained by using the reaction rate theory of Kramers in the high friction (diffusion limit) changed from a positive value to a negative value. The value of $E^{*}$ was also negative in the case of tripropionin, which is a poor solvent for PS and has a high viscosity. Therefore, we conclude that the negative $E^{*}$ does not depend on the quality of the solvent, i.e., whether the solvents are good or poor, but on the viscosity of the solvent. Then, we evaluated $E^{*}$ on the basis of Grote-Hynes' equation and of the Robinson's equation. The analysis by the Robinson's equation gave positive values of $E^{*}$. The anomaly of the activation energies of the local motions may be explained by the movement of the polymer chains in an ordered solvent of strong intermolecular interaction.

KEY WORDS Fluorescence Depolarization / Polystyrene / Diethylphthalate

/ High Viscosity Solvent / Kramers' Diffusion Limit / Polymer Chain Dynamics /
\end{abstract}

The dynamic property of polymer chains is a governing factor of the physical properties of polymers, and it is important to understand this character not only for application but also from a fundamental view point. Recently, the dynamic behavior of polymers has been extensively investigated on the molecular level. ${ }^{1-4}$ However, the relationship between the local motion and chemical structure of polymer chains is not fully understood. The elementary process in polymer chain dynamics corresponds to local conformation transitions, ${ }^{5-7}$ and this usually takes place in the order of nanoseconds or sub-nanoseconds.

To investigate the local motion there are

* To whom correspondence should be addressed. neutron scattering, ${ }^{8}$ NMR, ${ }^{9}$ ESR, ${ }^{1}$ dielectric relaxation, ${ }^{10,11}$ and fluorescence depolarization methods. ${ }^{12,13}$ In this work, we have investigated the local motion of the polymer chain in a dilute solution by the fluorescence depolarization method. The advantage of this method is that we can directly observe the orientational autocorrelation function. The disadvantage is that we need to introduce a fluorescent probe and in some cases the fluorescent probe may influence the behavior of its neighboring segment.

In this study, we investigated the local motion of polystyrene (PS) in high viscosity solvents by the subnanosecond time-resolved 
fluorescence depolarization method. ${ }^{14}$ We measured the mean relaxation time of local motions, and evaluated the activation energy of the conformation transitions.

\section{EXPERIMENTAL}

\section{Preparation of Polystyrene Sample}

Anthracene-labeled polystyrene (PS) chains were prepared by anionic polymerization initiated by $n$-butyllithium and the living end was deactivated by a bifunctional terminator 9,10-bis(bromomethyl)anthracene (Figure 1). The detailed preparative technique of this sample is described elsewhere. ${ }^{15}$ The prepared polymer was fractionated with GPC into two parts: the PS labeled at the middle of main chain, and that terminated by anthracene at the chain end. We employed the former for fluorescence depolarization measurements. The obtained PS has a $M_{w}$ of $9.7 \times 10^{4}, M_{w} / M_{n}$ of 1.05 , and racemo fraction of dyad of 0.54 .

\section{Solvents}

The solvents used for measurements are diethylphthalate (DEP, Wako Pure Chemical Industries), cyclohexanone (CHO, Nacalai Tesque), a mixed solvent of DEP : $\mathrm{CHO}=4: 1$ (vol), and a mixed solvent of DEP : $\mathrm{CHO}=3: 2$ (vol). Solvent viscosity and its activation energy were measured by capillary viscometry. The intrinsic viscosity for each solution was also measured by the viscometry. We evaluated the chain expansion factor, $\alpha_{\eta}$. The value is the ratio of the intrinsic viscosity of a solution to the intrinsic viscosity of the solution at the $\theta$ condition: in this study cyclohexane at $34.5^{\circ} \mathrm{C}$ was used.

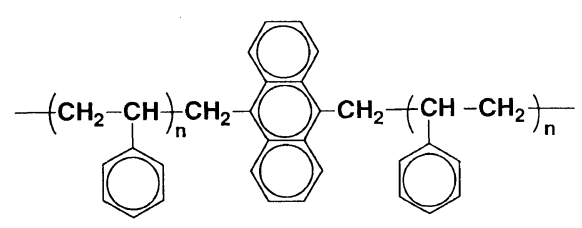

Figure 1. PS sample.
Measurements and Analysis of Anisotropy Ratio

The time-resolved fluorescence anisotropy ratio, $r(t)$, is defined as

$$
r(t)=\left(I_{\|}(t)-G I_{\perp}(t)\right) /\left(I_{\|}(t)+2 G I_{\perp}(t)\right)
$$

where $I_{\|}(t), I_{\perp}(t)$ are parallel and perpendicular component of fluorescence intensity, $G$ is the compensating factor $(G=1.0094)$. We measured $I_{\|}(t)$ and $I_{\perp}(t)$ alternatively by a computer control to avoid data distortions due to the time drift. The chromophore concentration of the solutions was kept less than $10^{-5} \mathrm{M}$, and the samples were degassed. The measurements were done by the single-photon counting system consisting of a time-to-amplitude converter (TAC, Ortec model 457), a discriminator (Ortec model 436), a constant fraction discriminator (Ortec model 583), and a multichannel analyzer (Norland Ino-Tech 5300). A diode laser PLP-01 (Hamamatsu Photonics, wavelength: $411 \mathrm{~nm}$, FWHM: $c a$. $50 \mathrm{ps}$ ) was used as a light source and a multichannel plate-photomultiplier tube as a detector. The repetition rate of this diode laser was $1 \mathrm{MHz}$, and we used the single-photoncounting system in the mode that the start and stop of TAC were reversed. The excitation light was vertically polarized. For excitation, we used a cutoff filter SC-41, and for observation the filters V-42 and Y-44 (HOYA). FWHM of the total instrumental function was $c a .300 \mathrm{ps}$. We convoluted eq 2 with the instrumental function to analyze $r(t)$, and fitted eq 2 to the experimental anisotropy data obtained from eq 1 by the method of nonlinear-least-squares. ${ }^{16}$

$$
\begin{aligned}
r(t)= & r_{0}\left[x \exp \left(-t / T_{1}\right)\right. \\
& \left.+(1-x) \exp \left(-t / T_{2}\right)\right]
\end{aligned}
$$

Equation 2 is empirical, but it represents the experimental data quite well. The mean relaxation time $T_{m}$ is defined by eq 3 , and this was calculated by eq 4 .

$$
T_{\mathrm{m}}=r_{0}{ }^{-1} \int_{0}^{\infty} r(t) \mathrm{d} t
$$




$$
T_{\mathrm{m}}=x T_{1}+(1-x) T_{2}
$$

\section{RESULTS AND DISCUSSION}

\section{Activation Energy of Solvent Viscosity and PS Chain Expansion}

Table I shows the solvent viscosities of tripropionin and two mixed solvents of DEP and $\mathrm{CHO}, \eta$, the activation energies of these solvent viscosities, $E_{\eta}$, and the chain expansion factors of PS chain, $\alpha_{\eta}$. DEP has a high viscosity and the activation energy of viscosity $E_{\eta}$ also has a high value of $6.3 \mathrm{kcal} \mathrm{mol}^{-1}$. The chain expansion factor shows that DEP, $\mathrm{CHO}$, and their mixed solvents are good solvents for PS, while tripropionin is a poor solvent with a high viscosity.

\section{Apparent Activation Energy of Local Polymer Motion}

Evaluation of $E^{*}$ by the Theory of Kramers' Diffusion Limit. We first evaluated the apparent activation energy of local motion $E^{*}$ by the theory of Kramers' diffusion limit. ${ }^{5,17}$ When a particle with a frictional coefficient $\zeta$ passes over an energy barrier with the height $E^{*}$, the velocity coefficient $k$ is presented by eq 5 ,

$$
k \propto \zeta^{-1} \exp \left(-E^{*} / R T\right)
$$

where $R$ is the gas constant and $T$ is absolute temperature. $T_{\mathrm{m}}$ is proportional to the reciprocal of $k$ and if we employ the Stokes law $\zeta \propto \eta$, we find

$$
T_{\mathrm{m}}=A \eta \exp \left(E^{*} / R T\right)
$$

Table I. Solvent viscosities at $20^{\circ} \mathrm{C}$, activation energies and chain expansion factor $\alpha_{\eta}$ for PS at $34.5^{\circ} \mathrm{C}$

\begin{tabular}{cccc}
\hline Solvent & $\eta / \mathrm{cP}$ & $E_{\eta} / \mathrm{kcal} \mathrm{mol}^{-1}$ & $\alpha_{\eta}$ \\
\hline Tripropionin & 7.63 & 6.5 & 0.929 \\
DEP : CHO & & & \\
$5: 0$ & 13.4 & 6.3 & 1.10 \\
$4: 1$ & 7.75 & 5.3 & 1.12 \\
$3: 2$ & 4.98 & 4.6 & 1.13 \\
$0: 5$ & 2.20 & 3.3 & 1.17 \\
\hline
\end{tabular}

Therefore we can obtain the apparent activation energy of the local motion of polymer $E^{*}$ directly from the slope of the plot of $\ln \left(T_{\mathrm{m}} / \eta\right)$ vs. $1 / T$ as can be seen from eq 7 .

$$
T_{\mathrm{m}} / \eta=A \exp \left(E^{*} / R T\right)
$$

Figure $2 \mathrm{a}-\mathrm{d}$ show the plots of $\ln \left(T_{\mathrm{m}} / \eta\right) v s$. $1 / T$. In Figure 2 the mixed ratio of DEP-CHO was varied from $0: 5$ (a), $3: 2$ (b), $4: 1$ (c), and 5:0 (d). For CHO which is a good solvent with a relatively low viscosity, we obtained the positive $E^{*}$ value of $1.0 \mathrm{kcal} \mathrm{mol}^{-1}$. With the addition of more DEP which is a good solvent with a high viscosity, the slope changed from a positive value to a negative value as can be seen from Figure $2 \mathrm{a}-\mathrm{d}$. Table II shows the each activation energy $E^{*}$, which was determined by the slope in Figure 2.

Here we found that the apparent activation energy $E^{*}$ is the negative value for high viscosity solvents. Previously we obtained the same result of negative $E^{*}$ for tripropionin which is a poor solvent for PS with a high viscosity. From the present findings, we conclude that such a result does not depend on the solvent quality, i.e., whether the solution is good or poor, but on the solvent viscosity.

Evaluation of $E_{\mathrm{GH}}^{*}$ by the Equation Using Grote-Hynes Theory. ${ }^{18}$ Secondly we evaluated the activation energy of a polymer chain by eq 8 based on Grote-Hynes theory to clarify the anomalous activation energy $E^{*}$ obtained from Kramers' diffusion limit.

$$
T_{\mathrm{m}}=A^{\prime} \eta^{\alpha} \exp \left(E_{\mathrm{GH}}^{*} / R T\right) \quad(0<\alpha<1)
$$

Here, we represented an activation energy as $E_{\mathrm{GH}}^{*}$. Grote and Hynes ${ }^{18}$ extended Kramers' theory by including the frequency dependence of the friction. In high viscosity solvents, the shear viscosity of the solvent does not adequately represent the friction experienced by the solute, and Grote-Hynes theory takes into account this effect.

Fleming and co-workers ${ }^{19,20}$ found in their experimental results that the viscosity dependence is well described by $\eta^{\alpha}$. The power-law 


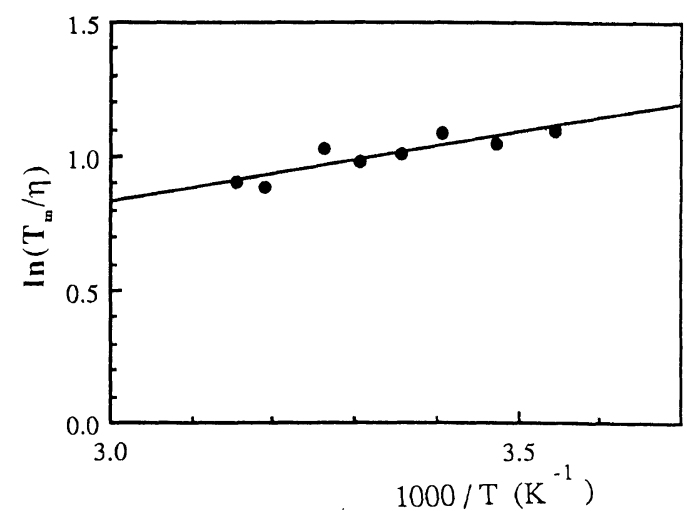

(a)

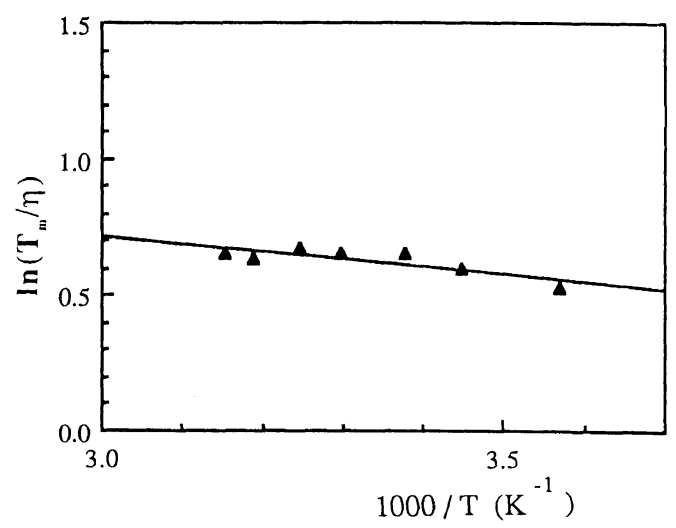

(c)

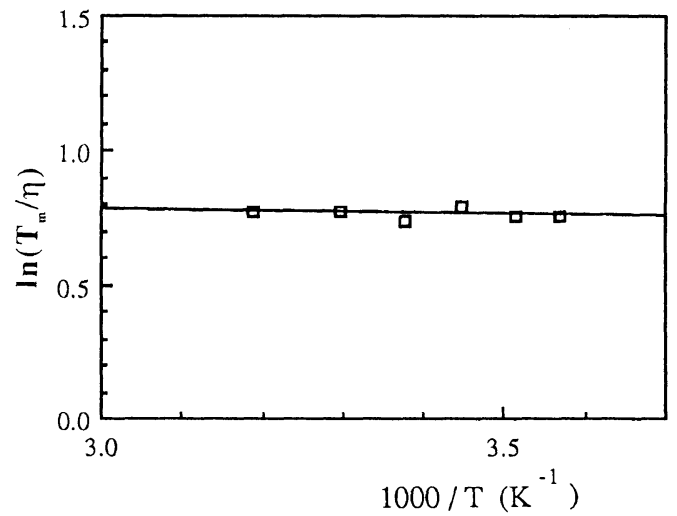

(b)

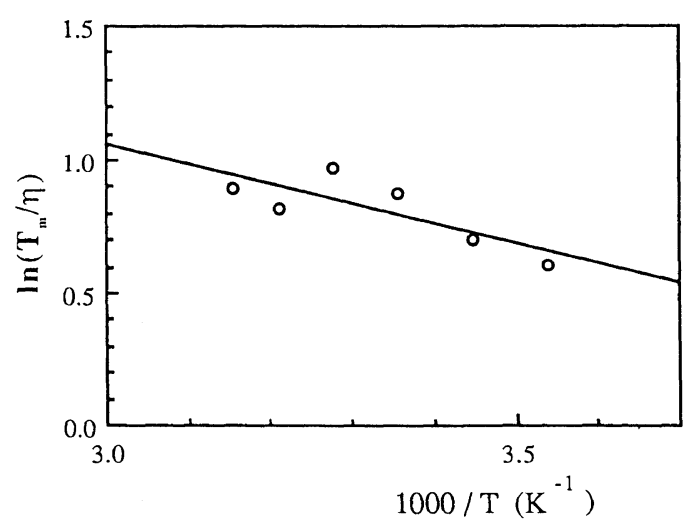

(d)

Figure 2. $\ln \left(T_{\mathrm{m}} / \eta\right)$ vs. $1 / T$ plots for PS solutions. Solvents are (a) CHO, (b) DEP-CHO $3: 2$, (c), DEP-CHO $4: 1$, and (d) DEP.

Table II. Activation energies evaluated by Kramers' equation for PS solutions

\begin{tabular}{cc}
\hline Solvent & $E^{*} / \mathrm{kcal} \mathrm{mol}^{-1}$ \\
\hline Tripropionin & -1.9 \\
DEP : CHO & \\
$5: 0$ & -1.4 \\
$4: 1$ & -0.5 \\
$3: 2$ & 0.0 \\
$0: 5$ & 1.0
\end{tabular}

form was also used by other workers. ${ }^{21-23}$

Moreover, Bagchi and Oxtoby ${ }^{24}$ showed that the effective friction is indeed much smaller in the barrier region for high viscosity solvents than the zero frequency friction. They found that the exponent $\alpha$ depends on several parameters which are not anticipated in Kramers' diffusion theory, and showed that the Grote-Hynes theory with frequency dependent friction can explain the experimental results of Fleming and co-workers.

The assumption for the determination of $\alpha$ is that $\alpha$ is a constant value independent of both solvent and temperature. When we make $\log -\log$ plots of $T_{\mathrm{m}}$ vs. $\eta$ based on this assumption, we can obtain the value of $\alpha$ from the slope. In this study, we obtained the $\alpha$ value of 0.79 at $25^{\circ} \mathrm{C}$. With $\alpha=0.79$ we determined the apparent activation energy of local motion, $E_{\mathrm{GH}}^{*}$. Table III shows $E_{\mathrm{GH}}^{*}$ determined by this procedure. $E^{*}$ is still negative for the 
Table III. Activation energies evaluated by Grote-Hynes' equation for PS solutions

\begin{tabular}{cc}
\hline Solvent & $E_{\mathrm{GH}}^{*} / \mathrm{kcal} \mathrm{mol}^{-1}$ \\
\hline DEP $:$ CH & \\
$5: 0$ & -0.1 \\
$4: 1$ & 0.6 \\
$3: 2$ & 1.0 \\
$0: 5$ & 1.7 \\
\hline
\end{tabular}

highest viscosity solvent. That is, $E^{*}$ depended on the kind of solvent. This means that the treatment of solvent viscosity in eq 8 is not adequate for the evaluation of the local motions of polymer chains and that the assumption of $\alpha=$ constant is not valid.

Evaluation of $E_{\mathrm{R}}^{*}$ by the Equation of Robinson. ${ }^{22,25}$ We also considered a different approach in order to evaluate the correct value for an activation energy. As for photoisomerization of stilbene, the rate $k_{\text {iso }}$ is commonly expressed by eq 9 .

$$
k_{\text {iso }}=F(\zeta) \exp \left(-E_{0} / R T\right)
$$

where $F(\zeta)$ is a function of friction, $\zeta$, and $E_{0}$ is an activation energy. Conventionally, the solvent friction is taken to be proportional to the solvent shear viscosity. However, Velsko et al. ${ }^{19}$ and Robinson et al. showed that the relation $\zeta \propto \eta$ is inadequate in high viscosity solvents. Robinson proposed eq 10 which presents the relation between a friction $\zeta$ and a viscosity $\eta$.

$$
F(\zeta)=A_{1}\left[1-1 /\left(A_{2} / \eta+A_{3}\right)\right]^{1 / 2}
$$

We derived eq 11 using eq 10 to extend the utility of Kramers theory. ${ }^{17}$

$$
T_{\mathrm{m}}=A_{1}^{\prime}\left[1-1 /\left(A_{2} / \eta+A_{3}\right)\right]^{-1 / 2} \exp \left(E_{\mathrm{R}}^{*} / R T\right)
$$

In eq $11 A_{1}^{\prime}, A_{2}$, and $A_{3}$ are adjustable parameters, and we made the plot of $T_{\mathrm{m}} v s . \eta$ in each solution at $13^{\circ} \mathrm{C}$ to obtain these values. The assumption for the determination of the parameters is that these parameters are independent of both solvent and temperature.
Table IV. Activation energies evaluated by Robinson's equation for PS solutions

\begin{tabular}{cc}
\hline Solvent & $E_{\mathrm{R}}^{*} / \mathrm{kcal} \mathrm{mol}^{-1}$ \\
\hline DEP : CH & \\
$5: 0$ & 1.9 \\
$4: 1$ & 2.0 \\
$3: 2$ & 2.2 \\
$0: 5$ & 2.3 \\
\hline
\end{tabular}

The parameters, $A_{2}$ and $A_{3}$, were evaluated by fitting eq 11 to these experimental data by the method of nonlinear-least-squares, where the initial setting values of $A_{2}$ and $A_{3}$ were 0.1 and 1 ( $A_{3} \geqq 1$, by definition). Then, we could determine the parameter values, $\dot{A}_{2}: 0.0286 \mathrm{cP}$, $A_{3}: 1.000$, when a curve fully fitted to the data in the whole range from the low viscosity solvents to the high viscosity ones.

Robinson et $a .^{25}$ reported that this expression also includes a frequency dependence, and this form of the friction fits both experimental data and molecular dynamics simulation quite well. They measured the isomerization rates for the various systems. For example, in the system of the excited state of 3,3'-diethyloxadicarbocyanine iodide in alcohols, where the alcohols were used in the viscosity range $0.2-14 \mathrm{cP}$, the parameter values were $A_{2}$ is $0.0182 \mathrm{cP}$ and $A_{3}$ is 1.001 . We also obtained similar values as shown above.

We calculated the activation energies using eq 11 and the parameters. Table IV shows the results. The activation energy $E_{\mathrm{R}}^{*}$ was 1.9 $2.3 \mathrm{kcal} \mathrm{mol}^{-1}$ for all four samples. This indicates that in a high viscosity solvent such as DEP we can obtain an appropriate positive value of $E_{\mathrm{R}}^{*}$ not by Kramers theory and Grote-Hynes theory, but by the consideration of a solvent friction using Robinson's equation. In the case of high viscosity solvents, it is important how the friction for a polymer local motion is evaluated. The treatment of the friction as a function of a viscosity using eq 8 (Grote-Hynes' equation; $\alpha=0.79$ ) is not still adequate in a high viscosity range, and the 
estimation of the friction using eq 11 (Robinson's equation) is more adequate, i.e., in higher viscosity, the evaluation by the latter shows a weaker friction effect for a local motion of polymer chain than that by the former.

We compared this value with that reported by others. Ediger et al. $^{26}$ studied the local motion of PS in various dilute solutions by fluorescence depolarization. They used the Generalized Diffusion and Loss (GDL) model for an analysis different from ours. According to their report, the complete decay of the anisotropy was not observed in high viscosity solvents above $4 \mathrm{cP}$, and the GDL model did not provide an ideal fit to their data. They made a reasonable guess about the shape of the correlation function in order to estimate the average relaxation times. They used an average of the $\tau_{2} / \tau_{1}$ values obtained from the data which decayed to $<0.05$ to constrain the shape of the correlation function for the case of the solvents above $4 \mathrm{cP}$. The average activation energy obtained in good solvents is $2.6 \pm 0.7 \mathrm{kcal} \mathrm{mol}^{-1}$. We consider that this value is similar to ours obtained in a series of DEP-CHO system.

Furthermore, we can also compare this value with the result obtained in good solvents for PS. Here the average activation energy which was obtained from the solvents of low viscosities below $1 \mathrm{cP}$, was $c a$. $1.7 \mathrm{kcal} \mathrm{mol}^{-1} \cdot{ }^{27}$ We consider that the activation energy of $c a$. $2 \mathrm{kcal} \mathrm{mol}^{-1}$ obtained in this paper is a reasonable value as a height of an internal barrier of $\mathrm{C}-\mathrm{C}$ bonds.

Structure of High Viscosity Solvents. We discussed three treatments of $E^{*}$ in high viscosity solvents. The former two treatments give unrealistic results and the third gives the positive activation energies. The previous work $^{28-32}$ by dielectric relaxation demonstrated that the relaxation time for the liquid glycerides shows a wide distribution. For example, Khudayarov et al. $^{28}$ reported that the distribution of relaxation times in glycerides results from the orderliness of liquids by inter- molecular interaction. Moreover, McDuffie and Litovitz ${ }^{29}$ reported that in associated solutions there is a significant difference between the temperature dependence of the viscosity and that of relaxation time measured by dielectric relaxation, but at lower temperatures the temperature dependence was the same. Furthermore, they reported that a structural group or a cluster exists in the liquid state, and that the group size decreases with increasing temperature with only a small group of molecules remaining at high temperatures.

Therefore, for evaluation of the dynamics of the middle segments of polymer chain in high viscosity solvents, the equation of Kramers' diffusion limit is not appropriate. In this case of the middle of PS chain, the differences between macro-viscosity and micro-viscosity become pronounced above $3 \mathrm{cP}$ of solvent viscosity, and the friction between a polymer chain and solvent molecules is not proportional to macro-viscosity. Probably the friction between a polymer chain and solvent molecules differ in the high viscosity solvents, depending on the kind of polymers and motional modes, e.g., PS or poly(methyl methacrylate), and the middle part or an end of a chain. A similar anomaly of polymer chain dynamics in high viscosity solvents has also been reported by other workers. ${ }^{33-36}$ For example, Ediger et $a l .{ }^{33}$ reported that the theory of Kramers' diffusion limit gives a negative value for an activation energy of polyisoprene in dioctylphthalate.

The intermolecular interaction (dipole-dipole interaction) in ester groups is considered to form a network structure of the solvent and make the viscosity of solvent high: then under such a circumstance, the middle part of a polymer chain moves in such an ordered solvent and simple application of Kramers' diffusion limit gives an erroneous activation energy. That is, under such a condition, bulk viscosity and local friction can operate over different length scales and an effective viscosity for a local segment must be taken into 
consideration even in low molecular weight solvents with high viscosities. ${ }^{37}$

\section{CONCLUSION}

We have investigated the local motion of PS chains in DEP-CHO mixed solvents by the fluorescence depolarization method. In high viscous solvents we obtained an unrealistic negative activation energy by the analysis using Kramers' diffusion limit equation and GroteHynes' equation. This negative activation energy is not related to whether the solvent is good or poor. As an alternative treatment, Robinson's equation was used for evaluation of activation energies and then, $E_{\mathrm{R}}^{*}$ of about $2 \mathrm{kcal} \mathrm{mol}^{-1}$ were obtained. We proposed that in high viscosity solvents polymer chains move in a network structure of solvents and give a lower activation energy than that of the solvent viscosity.

Acknowledgments. We are indebted to Dr. Takashi Sasaki, Fukui University, for the preparation of the anthracene-labeled PS. The present work was supported by a Grant-in-Aid for Scientific Research (No. 62470094) from the Ministry of Education, Science, and Culture of Japan. Professor M. D. Ediger is acknowledged for helpful discussions.

\section{REFERENCES}

1. R. T. Bailey, A. M. North, and R. A. Pethrick, "Molecular Motion in High Polymers," Clarendon Press, Oxford, 1981.

2. M. Doi and S. F. Edwards, "The Theory of Polymer Dynamics," Oxford Science Publishers, Oxford, 1986.

3. M. Nagasawa, Ed., "Molecular Conformation and Dynamics of Macromolecules in Condensed Systems," Elsevier, Amsterdam, 1988.

4. M. Winnik, Ed., "Photophysical and Photochemical Tools in Polymer Science," D. Reidel Publishing Company, Dordrecht, 1986.

5. E. Helfand, J. Chem. Phys., 54, 4651 (1971).

6. E. Helfand, Z. R. Wasserman, and T. A. Weber, Macromolecules, 13, 526 (1980).

7. T. Yoshizaki, M. Fujii, and H. Yamakawa, J. Chem. Phys., 82, 1003 (1985).

8. J. S. Higgins and A. Maconnachie, "Methods of Experimental Physics,” Vol. 23, Part C, K. Sköld and
D. L. Price, Ed., Academic Press, London 1987, p 287.

9. S. Glowinkowski, D. J. Gisser, and M. D. Ediger, Macromolecules, 23, 3520 (1990).

10. a) S. Mashimo, Macromolecules, 9, 91 (1976). b) S. Mashimo, P. Winsor, R. H. Cole, K. Matsuo, and W. H. Stockmayer, Macromolecules, 19, 682 (1986).

11. K. Adachi, Macromolecules, 23, 1816 (1990).

12. T. Sasaki and M. Yamamoto, Macromolecules, 22, 4009 (1989).

13. M. D. Ediger, Ann. Rev. Phys. Chem., 42, 225 (1991).

14. D. V. O'Connor and D. Phillips, "Time-Correlated Single-Photon Counting," Academic Press, London, 1984.

15. S. Yokotsuka, Y. Okada, Y. Tojo, T. Sasaki, and M. Yamamoto, Polym. J., 23, 95 (1991).

16. T. Sasaki, M. Yamamoto, and Y. Nishijima, Macromolecules, 21, 610 (1988).

17. H. A. Kramers, Physica, 7, 284 (1940).

18. R. F. Grote and J. T. Hynes, J. Chem. Phys., 73, 2715 (1980).

19. S. P. Velsko, D. H. Waldeck, and G. R. Fleming, $J$. Chem. Phys., 78, 249 (1983).

20. S. H. Courtney and G. R. Fleming, J. Chem. Phys., 83, 215 (1985).

21. N. S. Park and D. H. Waldeck, J. Chem. Phys., 91, 943 (1984).

22. D. H. Waldeck, Chem. Rev., 91, 415 (1991).

23. G. Rothenberger, D. K. Negus, and R. M. Hochstrasser, J. Chem. Phys., 79, 5360 (1983).

24. B. Bagchi and D. W. Oxtoby, J. Chem. Phys., 78, 2735 (1983).

25. J. Lee, S.-B. Zhu, and G. W. Robinson, J. Phys. Chem., 91, 4273 (1987).

26. D. A. Waldow, M. D. Ediger, Y. Yamaguchi, Y. Matsushita, and I. Noda, Macromolecules, 24, 3147 (1991)

27. K. Ono, Y. Okada, and M. Yamamoto, to be submitted.

28. U. V. Khudayarov and V. N. Khudaiberdyev, Russian J. Phys. Chem., 64, 1015 (1990).

29. G. E. McDuffie, Jr. and T. A. Litovitz, J. Chem. Phys., 37, 1699 (1962).

30. A. M. Ras and P. Bordewijk, Recueil, 90, 1055 (1971).

31. E. Ikada and T. Watanabe, J. Phys. Chem., 78, 1078 (1974).

32. G. van der Zwan and J. T. Hynes, J. Phys. Chem., 89, 4181 (1985).

33. D. B. Adolf, M. D. Ediger, T. Kitano, and K. Ito, Macromolecules, 25, 867 (1992).

34. R. L. Morris, S. Amelar, and T. P. Lodge, J. Chem. Phys., 89, 6523 (1988).

35. E. D. von Meerwall, S. Amelar, M. A. Smeltzly, and T. P. Lodge, Macromolecules, 22, 295 (1989).

36. P. J. Hains and G. Williams, Polymer, 16, 725 (1975).

37. J. D. Ferry, "Viscoelastic Properties of Polymers," 3rd ed, John Wiley and Sons, Inc., New York, N.Y., 1980, Chapter 12, p 329. 